\title{
Editorial: Nonlinear Structured Materials
}

\author{
Giles Hunt ${ }^{1,2 \star}$, Rainer Groh ${ }^{2}$, Tim Dodwell ${ }^{3}$ and Katia Bertoldi ${ }^{4}$ \\ ${ }^{1}$ Department of Mechanical Engineering, University of Bath, Bath, United Kingdom, ${ }^{2}$ Faculty of Engineering, University of \\ Bristol, Bristol, United Kingdom, ${ }^{3}$ College of Engineering, Mathematics and Physical Sciences, University of Exeter, Exeter, \\ United Kingdom, ${ }^{4}$ School of Engineering and Applied Sciences, Harvard University, Cambridge, MA, United States
}

Keywords: bistability and multistability, bifurcation, numerical modeling, structural design, additive manufacturing, lattice structure

\section{Editorial on the Research Topic}

\section{Nonlinear Structured Materials}

This collection of papers draws attention to a number of different circumstances where the global response of a macro-sized sample of complex material is governed at least partially by nonlinear structural effects on a finite micro-scale. The generic nature and wide applicability of this kind of behavior is nicely illustrated both by the variety of backgrounds from which the five contributions have been drawn, and the differing reasons for conducting the studies in the first place. We have contributions ranging from the physics of crystalline layered solids, through structural problems of analysis and design on the macroscale and continuing into large-scale modeling as found for example in applications to structural geology. Analysis, numerical modeling, structural design and novel manufacturing processes are all part of the picture, appearing under the banner of Nonlinear Structured Materials.

We first illustrate the overall picture by considering the paper by Wadee et al. This takes

OPEN ACCESS

Edited and reviewed by:

John L. Provis,

The University of Sheffield,

United Kingdom

*Correspondence:

Giles Hunt

g.w.hunt@bath.ac.uk

Specialty section:

This article was submitted to

Structural Materials,

a section of the journal

Frontiers in Materials

Received: 11 February 2021

Accepted: 25 February 2021

Published: 19 March 2021

Citation:

Hunt G, Groh R, Dodwell T and Bertoldi K (2021) Editorial: Nonlinear

Structured Materials.

Front. Mater. 8:666990.

doi: 10.3389/fmats.2021.666990 the well-known structural configuration of sandwich structures with lattice cores and focusses specifically on the design of the core structure to enhance damage tolerance and blast resistance. By forcing the cellular material in certain locations in the core to transition between conventional and auxetic phases, the overall stiffness can be changed from high resistance to effectively zero, without effecting permanent material damage. The work opens up new possibilities for harnessing positive aspects of elastic instability behavior, whereby structured materials can be designed and constructed to be potentially repairable, and hence reusable, after a hazardous loading event. Additive manufacturing (AM) techniques are proposed to introduce such bespoke features.

A similar underlying design theme can be found in the paper by O'Donnell et al. This focusses on developing physically intuitive design methods for macro-sized structural systems to encourage the use of nonlinear structural (meta-)materials. A geometric exploration of the feasible configurations of multi-stable hexagonal lattices, exhibiting bistability at the local level, identifies how the complexity of the design space may be significantly reduced, thereby simplifying the structural design process. The design of nonlinear structured materials by embedding instabilities at the unit-cell level has found many applications in the literature. The contribution by O'Donnell et al., on the other hand, presents a hierarchy of nonlinear springs, with each spring capable of an arbitrary and tunable load-displacement response created through a helical lattice structure of anisotropic strips. Using a compact energetic description of the lattices, the activation energy is readily identified and minimized. It is demonstrated how such methods can be applied to more complex lattice systems, whilst retaining their advantageous simplicity.

Anisotropy is also exploited by Loukaides. Here the local structural architecture is represented by an anisotropic smeared-out approximation and is further simplified by assuming bending takes 
place without stretching. The paper focusses specifically on obtaining bistability on the global level, for applications such as morphing or valve control. Engineers often look toward the growing body of manufacturing processes to produce exotic structural behavior, but the opposite can also be true; structured materials can allow manufacturing processes to exceed their envisaged capabilities. It is shown how multistability can be used to expand the limiting dimensions of AM processes, by printing a compact geometry that can be deployed to a larger configuration. Similarly, the synergies between AM and multistable structures can facilitate the creation of functional parts, such as valves and encapsulation devices, within a single manufacturing operation.

In the paper by Tordesillas et al., the focus falls on modeling of natural complex materials like concrete and rock. It demonstrates that unique properties can emerge from coupled evolution of the preferred pathways for force and damage propagation, governed by an optimized force transmission process. In particular, in the pre-failure regime, the process is one that exploits inherent redundancies in transmission pathways, to redirect forces and thus take damage away from the bottleneck where failure ultimately forms. The paper is part 1 of a two-part body of work dedicated to the centenary of the publication of Griffith's theory of brittle fracture, with part 2 found in Tordesillas et al. (2020).

\section{REFERENCES}

Tordesillas, A., Kahagalage, S., Ras, C., Nitka, M., and Tejchman, J. (2020). Early prediction of macrocrack location in concrete, rocks and other granular composite materials. Sci. Rep. 10:20268. doi: 10.1038/s41598-020-76616-y

Conflict of Interest: The authors declare that the research was conducted in the absence of any commercial or financial relationships that could be construed as a potential conflict of interest.
The modeling theme continues into the contribution from Barsoum. Here it is shown that atomic layers in layered solids like mica, graphite or the MAX phases are, for the most part, subject to the same physics as other layered systems, be they playing cards, geological strata or paper stacks. When atomic layers are loaded edge-on they can buckle. For the most part this is constrained buckling and mediated by a new defect mechanism, labeled a ripplocation, in contrast to basal dislocation: the deformation of macroscopic systems is thus seen to be applicable on the atomic scale. The identification of ripplocations as a new micro-deformation mechanism in layered solids is noteworthy since it doubles the number of such known mechanisms.

The diverse nature of contributions in this collection is testament to the cross-disciplinary research activity at heart of nonlinear structured materials. Nevertheless, there is a common thread running through all contributions in that geometric and material nonlinearity, when properly understood and controlled, can be the source of great creativity in designing highperformance man-made materials.

\section{AUTHOR CONTRIBUTIONS}

All authors listed have made a substantial, direct and intellectual contribution to the work, and approved it for publication.

Copyright (C) 2021 Hunt, Groh, Dodwell and Bertoldi. This is an open-access article distributed under the terms of the Creative Commons Attribution License (CC BY). The use, distribution or reproduction in other forums is permitted, provided the original author(s) and the copyright owner(s) are credited and that the original publication in this journal is cited, in accordance with accepted academic practice. No use, distribution or reproduction is permitted which does not comply with these terms. 